PROCEEDINGS OF THE

AMERICAN MATHEMATICAL SOCIETY

Volume 138, Number 2, February 2010, Pages 695-701

S 0002-9939(09)10107-7

Article electronically published on October 7, 2009

\title{
ON THE JAMES AND VON NEUMANN-JORDAN CONSTANTS IN BANACH SPACES
}

\author{
FENGHUI WANG
}

(Communicated by Nigel J. Kalton)

\begin{abstract}
Recently Alonso, Martín and Papini conjectured that the value of the von Neumann-Jordan constant is less than or equal to that of the James constant. This paper presents an affirmative answer to such a conjecture. Moreover, we obtain a sharp estimate for the von Neumann-Jordan constant.
\end{abstract}

\section{INTRODUCTION}

Both the James constant $J(X)$ and the von Neumann-Jordan constant $C_{\mathrm{NJ}}(X)$ play an important role in the description of various geometric structures. It is therefore worthwhile to clarify the relation between them. Kato, Maligranda and Takahashi [13] are the first who discussed their relation by the following inequality:

$$
C_{\mathrm{NJ}}(X) \leq \frac{[J(X)]^{2}}{1+[J(X)-1]^{2}}
$$

Since then, many authors have conducted worthwhile research on improving the above estimate. In 2003 Maligranda [15] formulated the following conjecture:

$$
C_{\mathrm{NJ}}(X) \leq 1+[J(X)]^{2} / 4 \text {. }
$$

Later on, some weak inequalities were obtained by several authors (see e.g. Maligranda et al. [16, Saejung [17, Takahashi [18]).

Recently Alonso, Martín and Papini [2] presented an inequality:

$$
C_{\mathrm{NJ}}(X) \leq 2(1+J(X)-\sqrt{2 J(X)}),
$$

which is a strong improvement of Maligranda's conjecture. Wang and Pang [19] strengthened this inequality as

$$
C_{\mathrm{NJ}}(X) \leq J(X)+\sqrt{J(X)-1}\left(\sqrt{1+(1-\sqrt{J(X)-1})^{2}}-1\right),
$$

but it is weaker than the inequality $C_{\mathrm{NJ}}(X) \leq J(X)$, conjectured by Alonso, Martín and Papini [2].

Received by the editors February 28, 2009, and, in revised form, July 1, 2009

2000 Mathematics Subject Classification. Primary 46B20.

Key words and phrases. Modulus of convexity, James constant, Neumann-Jordan constant.

This work was supported by the Natural Science Foundation of the Department of Education, Henan (2008A110012), development programs in science and technology of Henan Province (092300410187), and the Youth Foundation of Luoyang Normal University (2008-QNJJ-011).

(C)2009 American Mathematical Society Reverts to public domain 28 years from publication 
This paper is devoted to an investigation of the relation between the James and von Neumann-Jordan constants. We first state an inequality concerning the constants $A_{2}(X)$ and $J(X)$, which enables us to clarify the relation between $J(X)$ and $J\left(X^{*}\right)$. Another inequality related to the constants $E(X)$ and $J(X)$ is also obtained. This allows us to get a better estimate for the von Neumann-Jordan constant.

\section{Definitions AND NOTAtion}

Let $X$ be a real Banach space with $\operatorname{dim} X \geq 2$ and denote by $S_{X}$ and $B_{X}$ the unit sphere and the unit ball, respectively. The James constant

$$
J(X)=\sup \left\{\|x+y\| \wedge\|x-y\|: x, y \in S_{X}\right\}
$$

and the von Neumann-Jordan constant

$$
C_{\mathrm{NJ}}(X)=\sup \left\{\frac{\|x+y\|^{2}+\|x-y\|^{2}}{2\left(\|x\|^{2}+\|y\|^{2}\right)}: x \in S_{X}, y \in B_{X}\right\}
$$

have been extensively studied. For more details, we refer to [4, 5, 6, 17, 10, 11, 12, $13,14,15,16,17,18,20,19$. We also need some other constants:

$$
\begin{aligned}
A_{2}(X) & =\sup \left\{\frac{\|x+y\|+\|x-y\|}{2}: x, y \in S_{X}\right\}, \\
E(X) & =\sup \left\{\|x+y\|^{2}+\|x-y\|^{2}: x, y \in S_{X}\right\}, \\
g(X) & =\inf \left\{\max (\|x+y\|,\|x-y\|): x, y \in S_{X}\right\} .
\end{aligned}
$$

The first constant was defined by Baronti, Casini and Papini [3], and the other two were defined by Gao [9, 10]. It is worthwhile to mention that $J(X) g(X)=2$ (see [10, 13]).

The modulus of convexity $\delta_{X}(\epsilon):[0,2] \rightarrow[0,1]$ is defined as

$$
\delta_{X}(\epsilon)=\inf \left\{1-\frac{\|x+y\|}{2}: x, y \in S_{X},\|x-y\| \geq \epsilon\right\} .
$$

Obviously, $\delta_{X}(\epsilon)$ is nondecreasing on [0,2]. Moreover, the function $\delta_{X}(\epsilon) / \epsilon$ is also nondecreasing on $(0,2]$ (see [8]). Recall that a Banach space $X$ is called uniformly nonsquare if for any $x, y \in S_{X}$ there exists a $\delta>0$ such that either $\|x-y\| / 2 \leq 1-\delta$, or $\|x+y\| / 2 \leq 1-\delta$. It is well known that $X$ is uniformly nonsquare if and only if $J(X)<2$. The equality

$$
J(X)=2\left[1-\delta_{X}(J(X))\right]
$$

holds whenever $X$ is uniformly nonsquare (see [4]).

For simplicity we shall respectively denote $A_{2}(X), E(X), g(X)$ and $J(X)$ by $A, E, g$ and $J$ if it is required.

\section{Some PRELIMinary estimates}

Let us first state an estimate of $A_{2}(X)$ in terms of $J(X)$. The first inequality between them was stated by Alonso and Llorens-Fuster 1 as

$$
A_{2}(X) \leq 1+\frac{J(X)}{2},
$$

which has been improved by Wang and Pang [19] as

$$
A_{2}(X) \leq 1+\sqrt{J(X)-1} .
$$


The following is a further improvement of the above.

Theorem 1. For any Banach space $X$,

$$
A_{2}(X) \leq \frac{3 J(X)-2}{J(X)} .
$$

Proof. We may assume that $X$ is uniformly nonsquare, since in the case $J=2$ the inequality (2) is trivial. To show (2), we consider two cases for any $x, y \in S_{X}$.

Case $1 . J \leq\|x-y\| \leq 2$. Let $\|x-y\|=\epsilon$. According to the monotonicity of the function $\delta_{X}(\epsilon) / \epsilon$, one gets

$$
\begin{aligned}
\|x+y\|+\|x-y\| & \leq \epsilon+2\left(1-\delta_{X}(\epsilon)\right) \leq \epsilon+2\left(1-\frac{\delta_{X}(J)}{J} \epsilon\right) \\
& =\epsilon+(2-(g-1) \epsilon) \leq \max _{J \leq \epsilon \leq 2}(2-g) \epsilon+2 \\
& =6-2 g,
\end{aligned}
$$

where the first equality follows from (11).

Case 2. $\|x-y\| \leq J$. If $\|x+y\| \leq J$, then $\|x+y\|+\|x-y\| \leq 2 J$. Since

$$
J+\frac{2}{J}-3=\frac{(J-2)(J-1)}{J} \leq 0,
$$

we have $J \leq 3-2 / J=3-g$ and

$$
\|x+y\|+\|x-y\| \leq 2 J \leq 6-2 g .
$$

Conversely, if $\|x+y\| \geq J$, let $\|x+y\|=\epsilon$. Then we get as in Case 1 that

$$
\|x+y\|+\|x-y\| \leq 6-2 g \text {. }
$$

Therefore, from both cases, we get (2).

Corollary 2. For any Banach space $X$,

$$
A_{2}(X)-J(X) \leq(\sqrt{2}-1)^{2} .
$$

Proof. According to (2), we have

$$
\begin{aligned}
A_{2}(X)-J(X) & \leq \frac{-J^{2}(X)+3 J(X)-2}{J(X)} \\
& \leq \max _{\sqrt{2} \leq t \leq 2} \frac{-t^{2}+3 t-2}{t}=(\sqrt{2}-1)^{2},
\end{aligned}
$$

which gives the result.

Kato, Maligranda and Takahashi [13] discussed the relation between $J(X)$ and $J\left(X^{*}\right)$ and proved the following inequality:

$$
2(J(X)-1) \leq J\left(X^{*}\right) \leq J(X) / 2+1 .
$$

This gives an answer to the question posed by Gao and Lau [11. Wang and Pang [19] improved this inequality as

$$
1+(J(X)-1)^{2} \leq J\left(X^{*}\right) \leq 1+\sqrt{J(X)-1} .
$$

Applying Theorem 1, we can give a further improvement of the above estimates. 
Theorem 3. For any Banach space $X$,

$$
\frac{2}{3-J(X)} \leq J\left(X^{*}\right) \leq \frac{3 J(X)-2}{J(X)} .
$$

Proof. It follows from Theorem 1 that

$$
\begin{aligned}
\frac{2}{3-J(X)} & \leq \frac{2}{3-A_{2}(X)}=\frac{2}{3-A_{2}\left(X^{*}\right)} \\
& \leq J\left(X^{*}\right) \leq A_{2}\left(X^{*}\right) \\
& =A_{2}(X) \leq 3-2 / J(X),
\end{aligned}
$$

where one should note that $A_{2}(X)=A_{2}\left(X^{*}\right)$ [3, Proposition 2.2].

Corollary 4. For any Banach space $X$,

$$
\left|J(X)-J\left(X^{*}\right)\right| \leq(\sqrt{2}-1)^{2} .
$$

Proof. From (3) it follows that

$$
\begin{aligned}
J(X)-J\left(X^{*}\right) & \leq J(X)-\max \left\{\sqrt{2}, \frac{2}{3-J(X)}\right\} \\
& =\min \left\{J(X)-\sqrt{2}, \frac{-J^{2}(X)+3 J(X)-2}{3-J(X)}\right\} \\
& \leq \max _{\sqrt{2} \leq t \leq 2} \min \{f(t), g(t)\} \\
& =(\sqrt{2}-1)^{2},
\end{aligned}
$$

where $f(t)=t-\sqrt{2}$ and $g(t)=\left(-t^{2}+3 t-2\right) /(3-t)$. On the other hand, also from (3), it follows that

$$
\begin{aligned}
J\left(X^{*}\right)-J(X) & \leq \frac{-J^{2}(X)+3 J(X)-2}{J(X)} \\
& \leq \max _{\sqrt{2} \leq t \leq 2} \frac{-t^{2}+3 t-2}{t}=(\sqrt{2}-1)^{2},
\end{aligned}
$$

which completes the proof.

Next let us turn to the constant $E(X)$. Alonso, Martín and Papini 2 proved that

$$
2 J^{2}(X) \leq E(X) \leq 4 J(X),
$$

which can be strengthened as follows.

Theorem 5. For any Banach space $X$,

$$
E(X) \leq \frac{4\left(J^{2}(X)+4(J(X)-1)^{2}\right)}{J^{2}(X)} .
$$

Proof. Since $J(X) g(X)=2$, it suffices to show that

$$
E(X) \leq 4\left(1+(2-g(X))^{2}\right) .
$$

Assume again that $X$ is uniformly nonsquare and consider two cases for any $x, y \in$ $S_{X}$. 
Case 1. $J \leq\|x-y\| \leq 2$. Let $\|x-y\|=\epsilon$. The monotonicity of $\delta_{X}(\epsilon) / \epsilon$ yields

$$
\begin{aligned}
\|x+y\|^{2}+\|x-y\|^{2} & \leq \epsilon^{2}+4\left(1-\delta_{X}(\epsilon)\right)^{2} \\
& \leq \epsilon^{2}+4\left(1-\frac{\delta_{X}(J)}{J} \epsilon\right)^{2} \\
& =\epsilon^{2}+(2-(g-1) \epsilon)^{2} .
\end{aligned}
$$

Since the function

$$
\varphi(\epsilon):=\epsilon^{2}+(2-(g-1) \epsilon)^{2}
$$

is increasing on $[J, 2]$, we have

$$
\|x+y\|^{2}+\|x-y\|^{2} \leq \max _{J \leq \epsilon \leq 2} \varphi(\epsilon)=4\left(1+(2-g)^{2}\right) .
$$

Case 2. $\|x-y\| \leq J$. If $\|x+y\| \leq J$, then $\|x+y\|^{2}+\|x-y\|^{2} \leq 2 J^{2}$. Note that

$$
J^{4}-8 J^{2}+8 J=J(J-2)(J+1+\sqrt{5})(J+1-\sqrt{5}) \leq 0,
$$

which implies that

$$
J^{2} \leq \frac{8 J(J-1)}{J^{2}} \leq \frac{2\left(J^{2}+4(J-1)^{2}\right)}{J^{2}}=2\left(1+(2-g)^{2}\right) .
$$

Consequently,

$$
\|x+y\|^{2}+\|x-y\|^{2} \leq 2 J^{2} \leq 4\left(1+(2-g)^{2}\right) .
$$

On the contrary, if $\|x+y\| \geq J$, let $\|x+y\|=\epsilon$. We get as in Case 1 that

$$
\|x+y\|^{2}+\|x-y\|^{2} \leq 4\left(1+(2-g)^{2}\right) .
$$

Therefore, from both cases, we get (5) .

Remark 1. It is easy to see that

$$
J^{3}-\left(J^{2}+4(J-1)^{2}\right)=(J-1)(J-2)^{2} \geq 0,
$$

from which we have

$$
\frac{J^{2}+4(J-1)^{2}}{J^{2}} \leq J
$$

So Theorem 5 improves the inequality (4).

\section{Estimate FOR THE VON NEUmann-Jordan CONSTANT}

Following the ideas in [17, 20], we can rewrite

$$
C_{\mathrm{NJ}}(X)=\sup \left\{C_{\mathrm{NJ}}(t, X): 0 \leq t \leq 1\right\},
$$

where

$$
C_{\mathrm{NJ}}(t, X):=\sup \left\{\frac{\|x+t y\|^{2}+\|x-t y\|^{2}}{2\left(1+t^{2}\right)}: x, y \in S_{X}\right\} .
$$

Now let us state the main results.

Theorem 6. For any Banach space $X$,

$$
C_{\mathrm{NJ}}(X) \leq 1+\frac{2(J(X)-1)}{\sqrt{J^{2}(X)+(2-J(X))^{2}}+2-J(X)} .
$$


Proof. Let $x, y \in S_{X}$ and observe first that for every $0 \leq t \leq 1$,

$$
\|x \pm t y\| \leq t\|x \pm y\|+(1-t) .
$$

This together with (2) and (5) yields

$$
\begin{aligned}
C_{\mathrm{NJ}}(t, X) & =\sup \left\{\frac{\|x+t y\|^{2}+\|x-t y\|^{2}}{2\left(1+t^{2}\right)}: x, y \in S_{X}\right\} \\
& \leq \frac{E t^{2}+4 A t(1-t)+2(1-t)^{2}}{2\left(1+t^{2}\right)} \\
& \leq 1+2(2-g) \frac{(1-g) t^{2}+t}{1+t^{2}}:=F(t) .
\end{aligned}
$$

Let $t_{0}=\sqrt{(g-1)^{2}+1}-(g-1)$. Then $t_{0} \in[0,1]$ satisfies the equation $(1-g) t+1=$ $\left(1+t^{2}\right) / 2$. It is not difficult to deduce that

$$
\begin{aligned}
C_{\mathrm{NJ}}(X) & \leq \max \{F(t): 0 \leq t \leq 1\}=F\left(t_{0}\right) \\
& =1+\frac{2-g}{\sqrt{1+(g-1)^{2}}+g-1} .
\end{aligned}
$$

Thus (6) follows from $J(X) g(X)=2$.

In the paper 2 Alonso, Martín and Papini posed two questions: (1) Does the inequality $C_{\mathrm{NJ}}(X) \leq J(X)$ hold for any space? (2) Does the identity $C_{\mathrm{NJ}}(X)=$ $J(X)$ hold only when both constants are equal to 2 ? By using Theorem 6 we can affirmatively answer these two questions.

Corollary 7. For any Banach space $X$,

$$
C_{\mathrm{NJ}}(X) \leq J(X) .
$$

Moreover the equality holds if and only if $X$ is not uniformly nonsquare.

Proof. Since $\sqrt{2} \leq J \leq 2$, one gets

$$
1+\frac{2(J-1)}{\sqrt{J^{2}+(2-J)^{2}}+2-J}-J=\frac{(J-1)\left(J-\sqrt{J^{2}+(2-J)^{2}}\right)}{\sqrt{J^{2}+(2-J)^{2}}+2-J} .
$$

It is not hard to see that the right side above is less than or equal to zero and the equality holds if and only if $J=2$, and thus the result follows.

\section{ACKNOWLEDGEMENT}

The author would like to thank the referee for informing him that the conjecture $C_{\mathrm{NJ}}(X) \leq J(X)$ was also proved very recently by Takahashi and Kato in the paper "A simple inequality for the von Neumann-Jordan and James constants of a Banach space", to appear in J. Math. Anal. Appl.

\section{REFERENCES}

[1] J. Alonso, E. Llorens-Fuster, Geometric mean and triangles inscribed in a semicircle in Banach spaces, J. Math. Anal. Appl. 340 (2008), 1271-1283. MR2390928 (2009b:46023)

[2] J. Alonso, P. Martín, P.L. Papini, Wheeling around von Neumann-Jordan constant in Banach spaces, Studia Math. 188 (2008), 135-150. MR2430999

[3] M. Baronti, E. Casini, P.L. Papini, Triangles inscribed in a semicircle, in Minkowski planes, and in normed spaces, J. Math. Anal. Appl. 252 (2000), 124-146. MR.1797848(2002a:46007)

[4] E. Casini, About some parameters of normed linear spaces, Accad. Naz. Lincei Rend. Cl. Sci. Fis. Mat. Natur. 80 (1986), 11-15. MR944367 (89h:46022) 
[5] J.A. Clarkson, The von Neumann-Jordan constant for the Lebesgue spaces, Ann. of Math. (2) 38 (1937), 114-115. MR.1503327

[6] S. Dhompongsa, A. Kaewkhao, S. Tasena, On a generalized James constant, J. Math. Anal. Appl. 285 (2003), 419-435. MR2005130 (2004f:46020)

[7] S. Dhompongsa, P. Piraisangjun, S. Saejung, Generalised Jordan-von Neumann constants and uniform normal structure, Bull. Austral. Math. Soc. 67 (2003), 225-240. MR:1972712 (2005c:46015)

[8] T. Figiel, On the moduli of convexity and smoothness, Studia Math. 56 (1976), 121-155. MR 0425581 (54:13535)

[9] J. Gao, A Pythagorean approach in Banach spaces, J. Inequal. Appl., Art. ID 94982 (2006), 1-11. MR2215461 (2007a:46011)

[10] J. Gao, K.S. Lau, On the geometry of spheres in normed linear spaces, J. Austral. Math. Soc. Ser. A 48 (1990), 101-112. MR.1026841 (91e:46025)

[11] J. Gao, K.S. Lau, On two classes of Banach spaces with uniform normal structure, Studia Math. 99 (1991), 41-56. MR.1120738 (92h:46017)

[12] A. Jiménez-Melado, E. Llorens-Fuster, S. Saejung, The von Neumann-Jordan constant, weak orthogonality and normal structure in Banach spaces, Proc. Amer. Math. Soc. 134 (2006), 355-364. MR2176002 (2006e:46020)

[13] M. Kato, L. Maligranda, Y. Takahashi, On James and Jordan-von Neumann constants and the normal structure coefficient of Banach spaces, Studia Math. 144 (2001), 275-295. MR:1829721 (2002k:46035)

[14] M. Kato, Y. Takahashi, On the von Neumann-Jordan constant for Banach spaces, Proc. Amer. Math. Soc. 125 (1997), 1055-1062. MR1371131 (97f:46017)

[15] L. Maligranda, On an estimate of the Jordan-von Neumann constant by the James constant, August 2003, 5 pages, manuscript.

[16] L. Maligranda, L.I. Nikolova, L.E. Persson, T. Zachariades, On n-th James and Khintchine constants of Banach spaces, Math. Inequal. Appl. 11 (2008), 1-22. MR2376255(2008k:46048)

[17] S. Saejung, On James and von Neumann-Jordan constants and sufficient conditions for the fixed point property, J. Math. Anal. Appl. 323 (2006), 1018-1024. MR2260161 (2007j:46028)

[18] Y. Takahashi, Some geometric constants of Banach spaces-a unified approach, Proc. Internat. Symposium Banach and Function Spaces II, Yokohama Publishers, Kitakyushu, Japan, 2007, 191-220. MR2428600

[19] F. Wang, B. Pang, Some inequalities concerning the James constant in Banach spaces, J. Math. Anal. Appl. 353 (2009), 305-310. MR2508868

[20] C. Yang, F. Wang, On a new geometric constant related to the von Neumann-Jordan constant, J. Math. Anal. Appl. 324 (2006), 555-565. MR2262491(2007h:46024)

Department of Mathematics, Luoyang Normal University, Luoyang 471022, People's Republic of China

E-mail address: wfenghui@gmail.com 